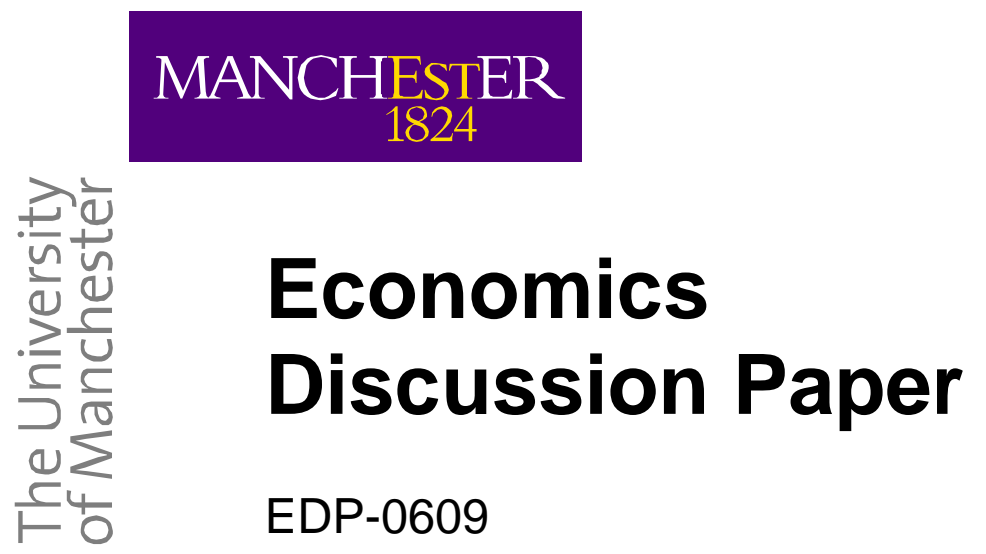

\title{
The Asymptotic Distribution of the F-Test Statistic for Individual Effects
}

by $\mathrm{C}$. Orme and $\mathrm{Y}$. Yamagata

May 2006 


\title{
The Asymptotic Distribution of the F-Test Statistic for Individual Effects*
}

\author{
Chris D. Orme \\ Economics, School of Social Sciences, University of Manchester \\ and \\ Takashi Yamagata \\ Faculty of Economics, University of Cambridge
}

May 10, 2006

\begin{abstract}
This paper employs first order asymptotic theory in order to establish the asymptotic distribution of the F-test statistic for fixed effects, under non-normality of the errors, when $N \rightarrow \infty$ (the number of crosssections) and $T$ is fixed (the number of time periods). Three theoretical results emerge: (i) the standard F-test procedure will still deliver asymptotically valid inferences; (ii) under local (pure) random effects, the F-test statistic is asymptotically proportional to the random effects test statistic and, therefore, both tests have the same asymptotic power; (iii) under local fixed, or random effects which are correlated with the regressors, the $F$-Test will have higher asymptotic power than the random effects test.
\end{abstract}

\section{Introduction}

Although there is a large statistics and econometrics literature on analysis of variance testing, it appears that results concerning the asymptotic properties of the commonly used F-test (for fixed effects) remain incomplete. First, virtually all standard econometric texts quote this test (and the statistic it is also reported by software packages such as STATA), but there is little or no comment on the finite sample importance of the normality assumption when $T$ (the number of time periods) is fixed, or small, relative to $N$ (the number

\footnotetext{
${ }^{*}$ We are grateful to Les Godfrey, Denise Osborn and Joao Santos-Silva for their helpful comments, and also to three referees whose constructive comments greatly improved the quality of this paper. The second author received financial support from the Graduate School of Social Sciences and the School of Economic Studies, University of Manchester. Address correspondence to: Takashi Yamagata, Faculty of Economics, University of Cambridge, Sidgwick Avenue, Cambridge CB3 9DE, UK; e-mail: ty228@econ.cam.ac.uk
} 
of cross-sections). An exception is Wooldridge (2002, p.274) who remarks that the asymptotic distribution (large $N$, fixed $T$ ) is unknown under nonnormality and to the best of our knowledge this remains true. Second, there appears to be no theoretical results available which explain the asymptotic relationship between the $F$-test statistic and the commonly used random effects test (RE-test) statistic; hereafter denoted $F_{N}$ and $R_{N}$, respectively.

The current paper addresses both these issues by deriving the limit distribution of the appropriately normalised and centred F-test statistic in the context of static panel data, under general non-normality of the errors, when $N \rightarrow \infty$ and $T$ fixed. Asymptotic local analysis is employed in order that the asymptotic sampling behaviour of this statistic can be analysed not only under the null (as a special case) but also more generally in the presence of individual effects. In this context, individual effects can be either fixed, random but correlated with the included regressors or purely random (uncorrelated with the included regressors). Three theoretical results emerge: (i) under the null (and local alternatives) the limit distribution of $\sqrt{N}\left(F_{N}-1\right)$ is normal, however the standard $F$-test procedure will still deliver asymptotically valid inferences; (ii) under local (pure) random effects, $F_{N}$ is asymptotically proportional to $R_{N}$ and, therefore, both tests have the same asymptotic power; (iii) under local fixed effects, or random effects which are correlated with the regressors, the $R E$-test procedure will have lower asymptotic power than the F-test procedure. Monte Carlo evidence is presented which supports these findings and suggests that the standard F-test procedure performs well, even in quite small samples, under nonnormality and is preferable to one which uses the approximating normal distribution in order to obtain finite sample critical values. Moreover, the $F$-test is found to be at least as powerful as the RE-test, as predicted by first order theory.

The plan of this paper is as follows. Section 2 introduces the notation and both test statistics. Assumptions are introduced in Section 3, justifying the ensuing asymptotic analysis which characterises the asymptotic behaviour of the F-test statistic, including its relationship with the $R E$-test statistic under the null and local alternatives. All proofs of the main propositions are relegated to the Appendix. Section 4 illustrates the main findings by reporting the results of a small Monte Carlo study and Section 5 concludes.

\section{The Notation, Model and Test Statistics}

\subsection{Notation and Model}

In the standard linear panel data model, individual effects for cross section $i$ are introduced as follows

$$
\mathbf{y}_{i}=\alpha_{i} \iota_{T}+\mathbf{X}_{i} \boldsymbol{\beta}_{1}+\mathbf{u}_{i}, \quad i=1, \ldots, N
$$


where $\mathbf{y}_{i}=\left(y_{i 1}, \ldots, y_{i T}\right)^{\prime}, \mathbf{u}_{i}=\left(u_{i 1}, \ldots, u_{i T}\right)^{\prime}, \iota_{T}$ is a $(T \times 1)$ vector of ones, and $\mathbf{X}_{i}=\left(\mathbf{x}_{i 1}, \ldots, \mathbf{x}_{i T}\right)^{\prime}$ a $(T \times K)$ matrix. The innovations, $u_{i t}$, have zero mean and finite constant variance, $0<\sigma^{2}<\infty$, for all $i$ and $t$, and the $\alpha_{i}$ are the individual effects. By stacking the $N$ equations of (1), the model for all individuals becomes

$$
\mathbf{y}=\mathbf{D} \boldsymbol{\alpha}+\mathbf{X} \boldsymbol{\beta}_{1}+\mathbf{u},
$$

where $\mathbf{y}=\left(\mathbf{y}_{1}^{\prime}, \ldots, \mathbf{y}_{N}^{\prime}\right)^{\prime}$ and $\mathbf{u}=\left(\mathbf{u}_{1}^{\prime}, \ldots, \mathbf{u}_{N}^{\prime}\right)^{\prime}$ are both $(N T \times 1)$ vectors, $\boldsymbol{\alpha}=\left(\alpha_{1}, \ldots, \alpha_{N}\right)^{\prime}$ is a $(N \times 1)$ vector, $\mathbf{D}=\left[\mathbf{I}_{N} \otimes \boldsymbol{\iota}_{T}\right]$ is a $(N T \times N)$ matrix, $\mathbf{X}=\left(\mathbf{X}_{1}^{\prime}, \ldots, \mathbf{X}_{N}^{\prime}\right)^{\prime}$ is a $(N T \times K)$ matrix, and $[\mathbf{D}, \mathbf{X}]$ has full column rank. Thus, for the purposes of the current exposition, $\mathbf{x}_{i t}=\left(x_{i t 1}, \ldots, x_{i t K}\right)^{\prime}$, $(K \times 1)$, contains no time invariant regressors, in particular a constant term corresponding to an overall intercept, since in the context of fixed effects this allows estimation of all the unknown regression parameters, $\left(\boldsymbol{\alpha}^{\prime}, \boldsymbol{\beta}_{1}^{\prime}\right)^{\prime}$, as follows.

In general, define the projection matrices, $\mathbf{P}_{\mathbf{B}}=\mathbf{B}\left(\mathbf{B}^{\prime} \mathbf{B}\right)^{-1} \mathbf{B}^{\prime}$ and $\mathbf{M}_{\mathbf{B}}=$ $\mathbf{I}_{N T}-\mathbf{P}_{\mathbf{B}}$, for any $(N T \times S)$ matrix $\mathbf{B}$ of full column rank, with $\tilde{\mathbf{B}}=\mathbf{M}_{\mathbf{D}} \mathbf{B}$ being the residual matrix from a multivariate least squares regression of $\mathbf{B}$ on $\mathbf{D}$ which is, of course, the within transformation. For example, conformably with $\mathbf{X}, \tilde{\mathbf{X}}=\left(\tilde{\mathbf{X}}_{1}^{\prime}, \ldots, \tilde{\mathbf{X}}_{N}\right)^{\prime}$, where $\tilde{\mathbf{X}}_{i}$ has rows $\left(\mathbf{x}_{i t}-\overline{\mathbf{x}}_{i}\right)^{\prime}, i=1, \ldots, N$, and $\overline{\mathbf{x}}_{i}=T^{-1} \sum_{t=1}^{T} \mathbf{x}_{i t}$ and similarly for $\tilde{\mathbf{y}}^{1}{ }^{1}$ Then the fixed effects (least squares dummy variable) estimator of $\boldsymbol{\beta}_{1}$ in (2) is given by

$$
\begin{aligned}
\tilde{\boldsymbol{\beta}}_{1} & =\left(\mathbf{X}^{\prime} \mathbf{M}_{\mathbf{D}} \mathbf{X}\right)^{-1} \mathbf{X}^{\prime} \mathbf{M}_{\mathbf{D}} \mathbf{Y} \\
& =\left(\tilde{\mathbf{X}}^{\prime} \tilde{\mathbf{X}}\right)^{-1} \tilde{\mathbf{X}}^{\prime} \tilde{\mathbf{y}}
\end{aligned}
$$

and the corresponding estimator of $\boldsymbol{\alpha}$ is

$$
\tilde{\boldsymbol{\alpha}}=\left(\mathbf{D}^{\prime} \mathbf{M}_{\mathbf{X}} \mathbf{D}\right)^{-1} \mathbf{D}^{\prime} \mathbf{M}_{\mathbf{X}} \mathbf{y} .
$$

The null model of no individual effects is the pooled regression model of

$$
\begin{aligned}
\mathbf{y} & =\beta_{0} \iota_{N T}+\mathbf{X} \boldsymbol{\beta}_{1}+\mathbf{u} \\
& =\mathbf{Z} \boldsymbol{\beta}+\mathbf{u}
\end{aligned}
$$

where $\mathbf{Z}=\left[\boldsymbol{\iota}_{N T}, \mathbf{X}\right]$, with $\mathbf{z}_{i t}=\left(1, x_{i t 1}, \ldots, x_{i t K}\right)^{\prime}$, which delivers the ordinary least squares estimator $\hat{\boldsymbol{\beta}}=\left(\hat{\beta}_{0}, \hat{\boldsymbol{\beta}}_{1}^{\prime}\right)^{\prime}=\left(\mathbf{Z}^{\prime} \mathbf{Z}\right)^{-1} \mathbf{Z}^{\prime} \mathbf{y}$.

The standard $F$-test for fixed effects requires estimation of both (2), treating the $\alpha_{i}$ as unknown parameters, and (5), whilst the standard $R E$-test only requires estimation of (5). In order to provide a framework in which to investigate the limiting behaviour of the F-test and RE-test statistics, under both fixed and random effects, the individual effects are assumed to have the form $\boldsymbol{\alpha}=\beta_{0} \boldsymbol{\iota}_{N}+\boldsymbol{\delta}, \boldsymbol{\delta}=\left(\delta_{1}, \ldots, \delta_{N}\right)^{\prime}$. Fixed effects correspond

\footnotetext{
${ }^{1}$ To see this, note that $\mathbf{P}_{D}=\left(\mathbf{I}_{N} \otimes T^{-1} \iota_{T} \iota_{T}^{\prime}\right)$.
} 
to the $\alpha_{i}, i=1, \ldots, N$, being fixed unknown parameters (or, equivalently, $\delta_{1} \equiv 0$ with $\beta_{0}$ and $\delta_{i}, i=2, \ldots, N$, being the fixed unknown parameters). The case of random effects is accommodated when the $\delta_{i}, i=1, \ldots, N$ are random variables. In general, equation (1) will be employed to characterise the data generation process with the restrictions of $H_{0}: \boldsymbol{\delta}=\delta_{1} \iota_{N}$ providing the null model of no individual effects (notice that $\boldsymbol{\delta}=\mathbf{0}$ belongs to this set of restrictions). Specifically, when considering the alternative of fixed effects the $(N-1)$ restrictions placed on $(2)$ are $H_{0}: \mathbf{H} \boldsymbol{\alpha}=\mathbf{0}$, where $\mathbf{H}=\left[\boldsymbol{\iota}_{N-1},-\mathbf{I}_{N-1}\right]$, whilst for random effects the null is $H_{0}: \operatorname{var}\left(\delta_{i}\right)=0$.

\subsection{The Test Statistics}

\subsubsection{The F-test statistic}

Consider, first, the standard F-test for fixed effects which is based on the statistic

$$
F_{N}=\frac{\left(R S S_{R}-R S S_{U}\right) /(N-1)}{R S S_{U} /(N(T-1)-K)},
$$

where $R S S_{R}=\hat{\mathbf{u}}^{\prime} \hat{\mathbf{u}}$ is the restricted sum of squares (from the pooled regression (5)) with $\hat{\mathbf{u}}=\mathbf{M}_{\mathbf{Z} \mathbf{y}}$, and $R S S_{U}=\tilde{\mathbf{u}}^{\prime} \tilde{\mathbf{u}}$ is the unrestricted sum of squares (from the fixed effects regression (2)) with $\tilde{\mathbf{u}}=\mathbf{M}_{\tilde{\mathbf{X}}} \tilde{\mathbf{y}}$, the residual vector from regressing $\tilde{\mathbf{y}}$ on $\tilde{\mathbf{X}}$. If normality and strong exogeneity were imposed such that, conditional on $\mathbf{X}, \mathbf{u}_{i} \sim N\left(\mathbf{0}, \sigma^{2} \mathbf{I}_{T}\right), i=1, \ldots, N$, then a standard F-test would be exact. However, as Wooldridge (2002, p274) remarks, "... the properties of this test as $N \rightarrow \infty$ with $T$ fixed are unknown without the normality assumption." 2

To appreciate the difficulties with standard asymptotic arguments, note that (6) can also be expressed as

$$
F_{N}=\frac{\sqrt{N} \tilde{\boldsymbol{\alpha}}^{\prime} \mathbf{H}^{\prime}\left[N^{-1} \mathbf{H}\left(\mathbf{D}^{\prime} \mathbf{M}_{\mathbf{X}} \mathbf{D}\right)^{-1} \mathbf{H}^{\prime}\right]^{-1} \sqrt{N} \mathbf{H} \tilde{\boldsymbol{\alpha}}}{(N-1) \tilde{\sigma}^{2}},
$$

where $\tilde{\sigma}^{2}=R S S_{U} /(N(T-1)-K)$. Since, in general, $\tilde{\sigma}^{2}$ is consistent for $\sigma^{2}$, the asymptotically validity of the $F$-test procedure would usually rest on $W_{N}=(N-1) F_{N}$ having a limiting $\chi^{2}$ distribution, in the absence of normality. However, this can not obtain with $T$ fixed and $N \rightarrow \infty$, since neither the dimension of $\mathbf{H} \tilde{\boldsymbol{\alpha}}$ nor $N^{-1} \mathbf{H}\left(\mathbf{D}^{\prime} \mathbf{M}_{\mathbf{X}} \mathbf{D}\right)^{-1} \mathbf{H}^{\prime}$ is bounded. In fact, it is shown below that, under non-normality, $\sqrt{N}\left(F_{N}-1\right)$ has a limit normal distribution so that $W_{N}=O_{p}(N)$.

\footnotetext{
${ }^{2}$ Interestingly, though, a recent result by Qin and Wan (2004, Theorem 3) implies that the null distribution of $(6)$ is still exactly central $F$ whenever the distribution of the error vector is a member of the class of spherically symmetric distributions; for example, a multivariate Student's $t$ distribution. However, the independence assumption employed in Section 3 only allows normality of the errors, within this class, and thus the approach of Qin and Wan (2004) offers no additional generality, as far as the distribution of (6) is concerned.
} 


\subsubsection{The Random Effects test statistic}

Define $v_{i}=\sum_{t=1}^{T} u_{i t}$ and $\mathbf{A}=\mathbf{A}^{\prime}=\iota_{T} \iota_{T}^{\prime}-\mathbf{I}_{T}$, so that $\sum_{i=1}^{N} v_{i}^{2}-\sum_{i=1}^{N} \sum_{t=1}^{T} u_{i t}^{2}=$ $\mathbf{u}^{\prime}\left(\mathbf{I}_{N} \otimes \mathbf{A}\right) \mathbf{u}$. Then, the RE-test statistic is constructed as

$$
R_{N}=\sqrt{\frac{N T}{2(T-1)}}\left[\frac{\hat{\mathbf{u}}^{\prime}\left(\mathbf{I}_{N} \otimes \mathbf{A}\right) \hat{\mathbf{u}}}{\hat{\mathbf{u}}^{\prime} \hat{\mathbf{u}}}\right]
$$

which has a limit standard normal distribution, as $N \rightarrow \infty$, under $H_{0}$. This statistic, and associated test procedure, is now well established in the literature; see, for example, Breusch and Pagan (1980), Chesher (1984) and Honda (1985). More generally, and under the assumption of homoskedasticity, $R_{N}$ can be regarded as test for serial correlation in the $u_{i t}$ as pointed out by Wooldridge (2002, p.265). However, the intimate (asymptotic) relationship between $F_{N}$ and $R_{N}$ has not previously been uncovered.

\section{Asymptotic Properties of $F_{N}$}

In this section we describe the properties of $F_{N}$, under both local fixed and random effects, by (i) deriving its asymptotic distribution, and (ii) establishing its asymptotic relationship with $R_{N}$. In the subsequent analysis, and without assuming normality, asymptotic theory is employed in which $N \rightarrow \infty$ and $T$ is fixed. To facilitate this, the following assumptions are made, which are of the sort found in, for example, White (2001, p.120):

\section{Assumption 1:}

(i) $\left\{\mathbf{X}_{i}, \mathbf{u}_{i}\right\}_{i=1}^{N}$ is an independent sequence;

(ii) $E\left(\mathbf{u}_{i} \mid \mathbf{X}_{i}\right)=\mathbf{0}$;

(iii) $E\left(\mathbf{u}_{i} \mathbf{u}_{i}^{\prime} \mid \mathbf{X}_{i}\right)=\sigma^{2} \mathbf{I}_{T}, 0<\sigma^{2}<\infty$.

\section{Assumption 2:}

(i) $E\left[\left|z_{i s k} u_{i t}\right|^{2+\eta}\right] \leq \Delta<\infty$ for some $\eta>0$, all $s, t=1, \ldots, T, k=$ $1, \ldots, K+1$, and all $i=1, \ldots, N$;

(ii) $E\left[\left|z_{i t k}\right|^{2+\eta}\right] \leq \Delta<\infty$ for some $\eta>0$, all $t=1, \ldots, T, k=1, \ldots, K+1$, and all $i=1, \ldots, N$;

(iii) $\mathbf{Q}_{N}=E\left(\mathbf{Z}^{\prime} \mathbf{Z} / N\right)$ is uniformly positive definite;

(iv) $\tilde{\mathbf{Q}}_{N}=E\left(\tilde{\mathbf{X}}^{\prime} \tilde{\mathbf{X}} / N\right)$ is uniformly positive definite.

\section{Assumption 3:}


(i) $E\left[u_{i t}^{2} u_{i s}^{2}\right]=\sigma^{4}$, for all $i$ and $t \neq s$;

(ii) $E\left[\left|u_{i t}\right|^{4+\eta}\right] \leq \Delta<\infty$ for some $\eta>0$, all $t=1, \ldots, T$, and all $i=$ $1, \ldots, N$.

\section{Assumption 4:}

(i) $\alpha_{i}=\beta_{0}+\frac{\delta_{i}}{N^{1 / 4}}, i=1, \ldots, N$;

(ii) $E\left|\delta_{i}\right|^{2+\eta} \leq \Delta<\infty$ for some $\eta>0$, and all $i=1, \ldots, N$;

(iii) $E\left[\boldsymbol{\delta}^{\prime} \boldsymbol{\delta} / N\right]$ is uniformly positive, where $\boldsymbol{\delta}^{\prime}=\left(\delta_{1}, \ldots, \delta_{N}\right)$.

Assumption 1 (i) reflects independent sampling of cross-section units and 1(ii) imposes a strong exogeneity assumption on $\mathbf{X}_{i}$, implying that $E\left(\tilde{\mathbf{X}}_{i}^{\prime} \mathbf{u}_{i}\right)=\mathbf{0}$ and thus ruling out (for example) lagged dependent variables. Moreover, by 1(iii), the elements of $\mathbf{u}_{i}$ are uncorrelated, but not necessarily independent. These familiar assumptions are found, for example, in Wooldridge (2002, Section 10). Assumption 2, together with Assumption 1 , delivers consistency and asymptotic normality of both the pooled and fixed effects least squares regression estimators ( $\hat{\boldsymbol{\beta}}$ and $\tilde{\boldsymbol{\beta}}_{1}$, respectively) and corresponding error variance estimators, and follow directly the approach of White (2001, Exercises 3.14, 5.12 and 6.2).

If Assumption 1 (ii) is weakened to $E\left(\mathbf{X}_{i}^{\prime} \mathbf{u}_{i}\right)=\mathbf{0}$, or even $E\left(\mathbf{x}_{i t} u_{i t}\right)=\mathbf{0}$ (zero contemporaneous correlation), $\tilde{\boldsymbol{\beta}}_{1}$ is not guaranteed to be consistent and, when it is inconsistent, the $F$-test statistic asymptotically invalid anyway, even under normality; for example, in the presence of lagged dependent variables - see the discussion in Wooldridge (2002, Sections 10.5 and 11.1). Assumption 3(i) ensures that elements of $\left\{u_{i t}^{2}\right\}_{t=1}^{T}$ are serially uncorrelated, and together with the uniform bound Assumption 3(ii), justifies the limit distribution obtained in Proposition 1 below. ${ }^{3}$ Finally, Assumption 4 characterises the alternative data generation process and permits the investigation of asymptotic power, under local individual effects, by restricting the test criteria under consideration to be $O_{p}(1)$ with well defined limit distributions. As well as fixed effects (with the $\delta_{i}$ being non-stochastic) it also accommodates local random effects by defining $\delta_{i}=\tau \varepsilon_{i}, \tau>0, i=1, \ldots, N$, where the $\varepsilon_{i}$ are independently distributed with zero mean and unit variance, and also distributed independently of $u_{i t}$ for all $i$, t. In this case, Assumptions 4 (ii) and (iii) ensure that $N^{-1} \boldsymbol{\delta}^{\prime} \boldsymbol{\delta}=\tau^{2} N^{-1} \sum_{i=1}^{N} \varepsilon_{i}^{2}=O_{p}(1)$, and converges to $\tau^{2}$ in probability. If the $\varepsilon_{i}$ are also distributed independently of $\mathbf{X}_{i}$, then we have "pure" random effects whilst if the $\varepsilon_{i}$ are correlated with $\mathbf{X}_{i}$ (but $\operatorname{var}\left(\varepsilon_{i} \mid \mathbf{X}_{i}\right)=\tau^{2}$ ) then we have "correlated" random effects. (As

\footnotetext{
${ }^{3}$ Assumptions 2(i), 3(i), 3(ii) could be replaced by $u_{i t}$ being iid and independent of $x_{i t k}$.
} 
pointed out by Wooldridge (2002, p.252), in microeconometric applications of panel data models with individual effects, the term fixed effect is generally used to mean correlated random effects, rather than $\alpha_{i}$ being strictly non-stochastic.)

Before proceeding with the formal asymptotic local analysis, it is worth reviewing the special case of normality and non-stochastic $\left(\alpha_{i} \iota_{T}, \mathbf{X}_{i}\right)$, with $\alpha_{i}=O(1)$. Under (2) with $\boldsymbol{\alpha}=\beta_{0} \iota_{N}+\boldsymbol{\delta}$, standard (finite sample) distribution theory shows that $(6)$ has a (singly) non-central $F$-distribution with non-centrality parameter which can be expressed as $\sigma^{-2} \boldsymbol{\delta}^{\prime} \mathbf{D}^{\prime} \mathbf{M}_{\mathbf{Z}} \mathbf{D} \boldsymbol{\delta}$, which is identically zero when $\boldsymbol{\delta}=\delta_{1} \iota_{N}$. (In the simple model of $y_{i t}=$ $\alpha_{i}+u_{i t}$, and writing $\alpha_{i}=\beta_{0}+\delta_{i}$, this non-centrality parameter is equal to $\frac{T}{\sigma^{2}} \sum_{i=1}^{N}\left(\delta_{i}-\bar{\delta}\right)^{2}$, where $\bar{\delta}=N^{-1} \sum_{i=1}^{N} \delta_{i}$; see, for example, Scheffe (1959).) From the exact properties of a non-central $F$-distribution, the approximate mean for large $N$ is $E\left[F_{N}\right] \cong 1+\sigma^{-2} \frac{\boldsymbol{\delta}^{\prime} \mathbf{D}^{\prime} \mathbf{M}_{\mathbf{Z}} \mathbf{D} \boldsymbol{\delta}}{N}$. The asymptotic results obtained in the next section, are consistent with this.

\subsection{The Asymptotic Distribution of the $F_{N}$}

The asymptotic distribution of $F_{N}$ under non-normality is given by following limiting distribution result.

Proposition 1 Under model (2) and Assumptions 1 to $4, \sqrt{N}\left(F_{N}-1\right)=$ $O_{p}(1)$, with

$$
\sqrt{N}\left(F_{N}-1\right)=\frac{1}{\sqrt{N}} \frac{\mathbf{u}^{\prime}\left(\mathbf{I}_{N} \otimes \mathbf{A}\right) \mathbf{u}}{(T-1) \sigma^{2}}+\frac{\lambda_{N}}{\sigma^{2}}+o_{p}(1)
$$

and

$$
\sqrt{N}\left(F_{N}-1\right)-\frac{\lambda_{N}}{\sigma^{2}} \stackrel{d}{\rightarrow} N\left(0, \frac{2 T}{T-1}\right)
$$

where

$$
\lambda_{N}=\frac{\boldsymbol{\delta}^{\prime} \mathbf{D}^{\prime} \mathbf{M}_{\mathbf{Z}} \mathbf{D} \boldsymbol{\delta}}{N} \geq 0
$$

Proof. See Appendix.

Notice that, by Assumptions 2(iii) and 4(iii), for example, $\lambda_{N}$ need not necessarily converge, but it will be $O(1)$, at most. In the special case no individual effects with $\boldsymbol{\delta}=\delta_{1} \iota_{N}, \lambda_{N} \equiv 0$, as it should be (this includes the case of $\boldsymbol{\delta}=\mathbf{0}$ ). Proposition 1 remains consistent with the limiting properties of the exact distributional results obtained under normality, and 
non-stochastic $\left(\alpha_{i} \iota_{T}, \mathbf{X}_{i}\right)$, insofar that the mean in the asymptotic (i.e., approximate large $N$ ) distribution of $F_{N}$, given by Proposition 1, is

$$
E\left[F_{N}\right] \cong 1+\sigma^{-2} \frac{\boldsymbol{\delta}^{\prime} \mathbf{D}^{\prime} \mathbf{M}_{\mathbf{Z}} \mathbf{D} \boldsymbol{\delta}}{N} .
$$

Under the null of $H_{0}: \boldsymbol{\delta}=\delta_{1} \iota_{N}$, Proposition 1 implies that asymptotically valid inferences can be obtained by employing $F_{N}^{*}=\sqrt{\frac{N(T-1)}{2 T}}\left(F_{N}-1\right)$ in conjunction with (one-sided, positive) critical values from a standard normal distribution; the test is one-sided since $\lambda_{N} \geq 0$. Nonetheless, the usual $F$-test procedure for fixed effects remains asymptotically valid in the sense that use of $F_{N}$ in conjunction with $F$ distribution critical values, will still provide asymptotically valid inferences, despite non-normality of the innovations, $u_{i t}$.

To see this, let $c_{N}$ be any chosen critical value from an $F$ distribution with $n_{1}$ and $n_{2}$ degrees of freedom, respectively, where $n_{1}=N-1$ and $n_{2}=N(T-1)-K$. The probability of not rejecting the null (the actual significance level) is given by $\operatorname{Pr}\left(F_{N} \leq c_{N}\right)=\operatorname{Pr}\left(F_{N}^{*} \leq c_{N}^{*}\right)$, where $c_{N}^{*}=$ $\sqrt{\frac{N(T-1)}{2 T}}\left(c_{N}-1\right)$. Then, if $\xi_{N}$ has an $F$ distribution with $n_{1}$ and $n_{2}$ degrees of freedom, the discrepancy between the actual and nominal significance level is, therefore,

$$
\begin{aligned}
\left|\operatorname{Pr}\left(F_{N} \leq c_{N}\right)-\operatorname{Pr}\left(\xi_{N} \leq c_{N}\right)\right|= & \left|\operatorname{Pr}\left(F_{N}^{*} \leq c_{N}^{*}\right)-\operatorname{Pr}\left(\xi_{N}^{*} \leq c_{N}^{*}\right)\right| \\
\leq & \sup _{z}\left|\operatorname{Pr}\left(F_{N}^{*} \leq z\right)-\operatorname{Pr}\left(\xi_{N}^{*} \leq z\right)\right| \\
\leq & \sup _{z}\left|\operatorname{Pr}\left(F_{N}^{*} \leq z\right)-\Phi(z)\right| \\
& +\sup _{z}\left|\operatorname{Pr}\left(\xi_{N}^{*} \leq z\right)-\Phi(z)\right|
\end{aligned}
$$

where $\xi_{N}^{*}=\sqrt{\frac{N(T-1)}{2 T}}\left(\xi_{N}-1\right)$ and $\Phi($.$) denotes the standard normal dis-$ tribution function. Now, by Proposition 1, the first term of the right hand side is $o(1)$. The second term is also $o(1)$ from elementary probability distribution theory. Therefore, using critical values from the $F$ distribution, in conjunction with the statistic $F_{N}$, becomes increasingly like using critical values from the $N\left(1, \frac{2 T}{N(T-1)}\right)$ distribution, which is the approximate, large $N$, distribution of $F_{N}$.

Under pure local random effects $\frac{\mathbf{X}^{\prime} \mathbf{D} \delta}{N}=\tau \frac{T}{N} \sum_{i=1}^{N} \varepsilon_{i} \overline{\mathbf{x}}_{i}=o_{p}(1)$, (since $\left.\operatorname{var}\left(\frac{1}{N} \sum_{i=1}^{N} \varepsilon_{i} \overline{\mathbf{x}}_{i}\right)=o(1)\right)$ so that $\lambda_{N}=\frac{\boldsymbol{\delta}^{\prime} \mathbf{D}^{\prime} \mathbf{D} \boldsymbol{\delta}}{N}=T \tau^{2} \frac{1}{N} \sum_{i=1}^{N} \varepsilon_{i}^{2} \stackrel{p}{\rightarrow} T \tau^{2}$. Then, with a slight adaptation of the proof, we obtain the following Corollary to Proposition 1: 
Corollary 1 Under the alternative of local random effects, and under the assumptions of Proposition 1,

$$
\sqrt{N}\left(F_{N}-1\right) \stackrel{d}{\rightarrow} N\left(\frac{T \tau^{2}}{\sigma^{2}}, \frac{2 T}{T-1}\right) .
$$

Therefore, the F-test will have non-trivial asymptotic local power against random effects. In fact, a stronger result will be established in Section 3.2 which shows that, under local "pure" random effects, the F-test statistic is proportional (asymptotically) to the RE-test statistic, and will thus posses the same asymptotic power. However, under local "correlated" random effects the $F$-test will possess higher asymptotic power than the RE-test.

\subsection{The Relationship with $R_{N}$}

Under the null of no individual effects, it is readily shown that

$$
\begin{aligned}
\frac{1}{\sqrt{N}}\left[\frac{\hat{\mathbf{u}}^{\prime}\left(\mathbf{I}_{N} \otimes \mathbf{A}\right) \hat{\mathbf{u}}}{\hat{\mathbf{u}}^{\prime} \hat{\mathbf{u}} / N T}\right] & =\frac{1}{\sqrt{N}}\left[\frac{\mathbf{u}^{\prime} \mathbf{M}_{\mathbf{Z}}\left(\mathbf{I}_{N} \otimes \mathbf{A}\right) \mathbf{M}_{\mathbf{Z}} \mathbf{u}}{\mathbf{u}^{\prime} \mathbf{M}_{\mathbf{Z}}^{\prime} \mathbf{u} / N T}\right] \\
& =\frac{1}{\sqrt{N}} \frac{\mathbf{u}^{\prime}\left(\mathbf{I}_{N} \otimes \mathbf{A}\right) \mathbf{u}}{\sigma^{2}}+o_{p}(1)
\end{aligned}
$$

using the fact that $N^{-1 / 2} \mathbf{u}^{\prime} \mathbf{M}_{\mathbf{Z}}\left(\mathbf{I}_{N} \otimes \mathbf{A}\right) \mathbf{M}_{\mathbf{Z}} \mathbf{u}=N^{-1 / 2} \mathbf{u}^{\prime}\left(\mathbf{I}_{N} \otimes \mathbf{A}\right) \mathbf{u}+$ $o_{p}(1)$ and $\mathbf{u}^{\prime} \mathbf{M}_{\mathbf{Z}} \mathbf{u} / N T \stackrel{p}{\rightarrow} \sigma^{2}$. Therefore, by Proposition 1 , the asymptotic standard normal RE-test statistic (8) can be expressed as

$$
R_{N}=\left\{\sqrt{\frac{T-1}{2 T}}\right\} \sqrt{N}\left[F_{N}-1\right]+o_{p}(1) .
$$

Extending this analysis, the following Proposition establishes the asymptotic relationship between the two test statistics, under local individual effects (fixed or random). Apart from being of some interest in its own right it also helps explains some of the Monte Carlo evidence previously reported by Moulton and Randolph (1989) and Baltagi et al (1992).

Proposition 2 Under model (2) and Assumptions 1 to 4,

$$
R_{N}=\left\{\sqrt{\frac{(T-1)}{2 T}}\right\} \sqrt{N}\left[F_{N}-1\right]-\sqrt{\frac{T}{2(T-1)}} \gamma_{N}+o_{p}(1)
$$

where

$$
\gamma_{N}=\frac{\boldsymbol{\delta}^{\prime} \mathbf{D}^{\prime} \mathbf{Z}\left(\mathbf{Z}^{\prime} \mathbf{Z}\right)^{-1}\left(\tilde{\mathbf{Z}} \tilde{\mathbf{Z}}^{\prime}\right)\left(\mathbf{Z}^{\prime} \mathbf{Z}\right)^{-1} \mathbf{Z}^{\prime} \mathbf{D} \boldsymbol{\delta}}{N} \geq 0
$$

and the limit distribution of $\sqrt{N}\left[F_{N}-1\right]$ is given by Proposition 1.

Proof. See Appendix. 
Again, $\gamma_{N}$ need not converge to a positive constant, but it is $O_{p}(1)$. As with Proposition $1, \gamma_{N} \equiv 0$ obtains under $H_{0}: \boldsymbol{\delta}=\delta_{1} \iota_{N}$, as it should, since $\left(\mathbf{Z}^{\prime} \mathbf{Z}\right)^{-1} \mathbf{Z}^{\prime} \mathbf{D} \boldsymbol{\delta}=\left(\delta_{1}, \mathbf{0}^{\prime}\right)^{\prime}$ and the top-left, $(1,1)$, element of $\tilde{\mathbf{Z}}^{\prime} \tilde{\mathbf{Z}}$ is 0 . Under the alternative of "pure" local random effects $\gamma_{N}=o_{p}(1)$ since $\frac{\mathbf{Z}^{\prime} \mathbf{D} \boldsymbol{\delta}}{N}=o_{p}(1)$, and we obtain the following Corollary:

Corollary 2 Under the alternative of local random effects, and under the assumptions of Proposition 1,

$$
\begin{aligned}
R_{N}= & \left\{\sqrt{\frac{(T-1)}{2 T}}\right\} \sqrt{N}\left[F_{N}-1\right]+o_{p}(1) \\
& \stackrel{d}{\rightarrow} N\left(\frac{\tau^{2}}{\sigma^{2}} \sqrt{\frac{T(T-1)}{2}}, 1\right) .
\end{aligned}
$$

Since $R_{N}$ and $F_{N}^{*}=\sqrt{\frac{N(T-1)}{2 T}}\left(F_{N}-1\right)$ are asymptotically equivalent, both the $R E$ and $F$-test procedures will have identical asymptotic power functions, under local random effects. This conclusion helps explain the results reported by Moulton and Randolph (1989) and Baltagi et al (1992), whose analyses (undertaken under the assumption normality) are consistent with the theoretical findings presented here. However, under local fixed effects or random effects which are correlated $\mathbf{X}_{i}$, the $F$-test can have greater asymptotic power. In particular, when individual effects are correlated with the mean values of the regressors, $\mathbf{Z}^{\prime} \mathbf{D} \boldsymbol{\delta}=T \sum_{i=1}^{N} \delta_{i} \overline{\mathbf{z}}_{i} \neq \mathbf{0}$ and is $O_{p}(1)$, implying $\gamma_{N}>0$ so that a test based on $R_{N}$ should have lower asymptotic local power than one based on $F_{N}$. This makes intuitive sense, since $F_{N}$ is designed to test for individual effects which are correlated with $\overline{\mathbf{z}}_{i}$, whereas $R_{N}$ is constructed on the assumption that the individual effects are uncorrelated with all regressor values. The importance of distinguishing between individual effects which are correlated or uncorrelated with regressors, rather than simply labelling them fixed or random, is discussed by Wooldridge (2002, Section 10.2).

In summary, the two propositions presented in this section suggest that, under in the absence of individual effects, applied workers can be fairly confident of the F-test being robust to non-normality. Moreover, under the alternative of local random effects, the $F$-test statistic will be asymptotically proportional to the $R E$-test statistic and, therefore, the two procedures will have the same asymptotic power. However, under local individual effects which are fixed/correlated with the regressors, the $F$-test should have the greater asymptotic power.

In the next section, the preceding analysis is supported by the results of a small Monte Carlo experiment which illustrates the asymptotic robustness of the F-test to non-normality and its power properties relative to the $R E$ test. 


\section{Monte Carlo Simulation}

In order to shed light on the relevance of the preceding asymptotic analysis for finite sample behaviour, this section reports the results of a small Monte Carlo experiment which investigates the sampling behaviour of $F_{N}, F_{N}^{*}$ and $R_{N}$ under a variety of error distributions using $N=20,50,100, T=5$. The null model employed is

$$
y_{i t}=\sum_{j=1}^{3} z_{i t, j} \beta_{j}+u_{i t}
$$

where $z_{i t, 1}=1, z_{i t, 2}$ is drawn from a uniform distribution on $(1,31)$ independently for $i$ and $t$, and $z_{i t, 3}$ is generated following Nerlove (1971), such that

$$
z_{i t, 3}=0.1 t+0.5 z_{i t-1,3}+v_{i t}
$$

where the value $z_{i 0,3}$ is chosen as $5+10 v_{i 0}$, and $v_{i t}$ is drawn from the uniform distribution on $(-0.5,0.5)$ independently for $i$ and $t$, in order to avoid any normality in regressors. These regressor values are held fixed over replications. Also, observe that the regression design is not quadratically balanced. ${ }^{4}$ Table 1 shows the largest value of $h_{s} / \bar{h}$, where $h_{s}$ is the $s^{\text {th }}$ diagonal elements of $\mathbf{P}_{\mathbf{Z}}, s=1,2, \ldots, N T$ and $\bar{h}$ is the average of $h_{s}$, and the number of leverage points, where $h_{s} / \bar{h}>2,{ }^{5}$ confirming that the regressors used are not quadratically balanced.

\section{[Table 1 about here]}

Under the null, both $F_{N}$ and $S_{N}$ have sampling distributions which are independent of the $\beta_{j}$ and $\sigma^{2}$. Therefore, without loss of generality, the coefficients are set as $\beta_{j}=1$ for $j=1,2,3$ and the error terms, $u_{i t}$, are all $\operatorname{iid}(0,1)$ in the experiments. They are drawn from the following distributions and standardised: (i) standard normal distribution $(S N)$; (ii) Student's $t$ distribution with 5 degrees of freedom $(t(5))$; (iii) uniform distribution over the unit interval $(U N)$; (iv) mixture normal distribution from either $N(-1,1)$ and $N(1,1)$ with equal probability of $0.5(M N)$; (v) log-normal distribution $(L N)$; and, (vi) chi-square distribution with 2 degrees of freedom $\left(\chi^{2}(2)\right)$.

The sampling behaviour of three tests are investigated using 5000 replications of sample data and employing a nominal $5 \%$ significance level based on the predictions of the asymptotic theory presented in Section $3 .{ }^{6}$ Specifically, the behaviour of the following three tests is investigated:

\footnotetext{
${ }^{4}$ The results of Ali and Sharma (1996) show that, with a quadratically balanced design, the effects of non-normality on the $F$-test for linear restrictions in the linear model is minimal. Hence the Monte Carlo design guards against that possibility.

${ }^{5}$ See Belsley et al. (1980) for the discussion of leverage points.

${ }^{6} \mathrm{~A}$ full set of results employing $1 \%, 5 \%$ and $10 \%$ nominal significance levels are available upon request.
} 
1. F-test: Reject $H_{0}$ if $F_{N}>c$, where $\operatorname{Pr}\left(\xi_{N}>c_{N}\right)=5 \%$, and $\xi_{N} \sim$ $F(N-1,4 N-2)$.

2. Standard Normal F-test: Reject $H_{0}$ if $F_{N}^{*}=\sqrt{\frac{2 N}{5}}\left(F_{N}-1\right)>1.645$.

3. Standard Normal RE-Test: Reject $H_{0}$ if $R_{N}>1.645$.

Table 2 shows the estimated significance levels for the tests based on $F_{N}$, $F_{N}^{*}$ and $R_{N}$, as described above, for all error distributions. ${ }^{7}$

\section{[Table 2 about here]}

The $F$-test will be exact under normality, since $F_{N}$ is an exact pivot, and the results reflect this. Under non-normality such a procedure is only asymptotically valid, although it performs well (in terms of agreement between estimated and nominal significance levels) for all designs. ${ }^{8}$ Similarly, the random effects test based on $R_{N}$ exhibits quite close agreement between nominal and estimated significance levels. These results support the asymptotic analysis of Section 3 and suggest that standard F-test procedure (as reported in standard econometric texts) remains robust to non-normality even in small samples. (Of course, since $F_{N}$ is asymptotically pivotal, the results of Beran (1988) imply that improved finite sample performance may be obtained through the use of bootstrap critical values; see Yamagata (2004) who provides evidence which confirms this.) On the other hand, the onesided test based on $F_{N}^{*}=\sqrt{\frac{2 N}{5}}\left(F_{N}-1\right)$ is typically over-sized and should not, therefore, be relied upon to provide asymptotically valid inferences, under the null.

We now turn to a comparison of power but, due its poor sampling behaviour under the null, we do not consider $F_{N}^{*}$ further. In order to save space, only results for errors drawn from a standard normal distribution, a mixture normal distribution and a chi-square distribution, as described above, are reported. Individual effects are introduced through $\alpha_{i}$, as described in equation (1), and constructed as:

$$
\alpha_{i}=\tau \omega_{i}
$$

\footnotetext{
${ }^{7}$ We also considered another test, based on a scaled version of $R_{N}$ and critical values from a $F$ distribution. However as this seems rather ad hoc and its sampling behaviour under the null was found to be extremely poor it is not considered further in this paper.

${ }^{8}$ With $L N$ errors at the $10 \%$ nominal significance level, not reported here, it appeared somewhat less satisfactory with $N=20$, although this problem disappeared when $N=50$. Under skewed $\chi^{2}(2)$ errors there is still very good agreement between estimated and nominal significance levels. However, the kurtosis is much less in this case (6) than for the log-normal errors (113.94), suggesting that finite sample behaviour is more sensitive to excess kurtosis as, for example, implied by the work of Box and Andersen (1955), but in the non-regression context.
} 
with

$$
\omega_{i}=g_{i}\left(\overline{\mathbf{z}}_{i}\right)+\varepsilon_{i},
$$

where $\tau$ is a constant to control the cross section variation of $\alpha_{i}$, the $\varepsilon_{i}$ are iid $N\left(0,1-R^{2}\right), g_{i}\left(\overline{\mathbf{z}}_{i}\right)=\sqrt{R^{2}}\left(\iota_{3}^{\prime} \overline{\mathbf{z}}_{i} / s\right)$ with $\boldsymbol{\iota}_{3}=(1,1,1)^{\prime}, s$ being the standard deviation of $\iota_{3}^{\prime} \overline{\mathbf{z}}_{i}$, and the $R^{2}$ is from the regression of (10). With this set up, the variance of $\omega_{i}$ is always unity across designs. The value of $R^{2}$ is set at either 0 or 0.5 ; the former to examine the individual effects which are uncorrelated with regressors, the latter to examine those which are correlated with regressors. To sum up, four experiments are conducted and within each experiment values of $\tau=0.05,0.1,0.2$ are considered, so as to provide a range of powers for the two test procedures.

Experiment FE 1

$R^{2}=0.5$, so that the $\varepsilon_{i}$ are drawn independently, once, from a $N(0,0.5)$ distribution, for $i=1, \ldots, N$, and then held fixed across all 5000 replications of sample data.

Experiment FE 2

$R^{2}=0.0$, so that the $\varepsilon_{i}$ are drawn independently, once, from a $N(0,1)$ distribution, for $i=1, \ldots, N$, and then held fixed across all 5000 replications of sample data.

Experiment RE 1

$R^{2}=0.5$, so that the $\varepsilon_{i}$ are drawn independently from a $N(0,0.5)$ distribution, for $i=1, \ldots, N$, and for each replication of sample data.

Experiment $R E 2$

$R^{2}=0.0$, so that the $\varepsilon_{i}$ are drawn independently from a $N(0,1)$ distribution, for $i=1, \ldots, N$, and for each replication of sample data.

\section{[Table 3 about here]}

Table 3 reports the results. The first two experiments, FE 1 and $F E 2$ correspond to "fixed" effects, with FE 1 generating correlation between the fixed effect and the regressor values. In $F E 2$ there is no such correlation. The second two experiments, $R E 1$ and $R E 2$ correspond to random effects, with non-zero correlation between the random effects and the regressors in $R E$ 1, but not in $R E$ 2. Consistent with Proposition 2, the rejection frequencies of $F$-test is higher than the corresponding rejection frequencies of the $R E$-test under experiments $F E 1$ and $R E 1$, but they are very similar under experiments $F E 2$ and $R E 2$ for all error specifications considered.

\section{Conclusions}

This paper has addressed an apparent gap in the econometrics literature - as identified, for example, by Wooldridge (2002) - by providing the asymptotic distribution of the standard F-test statistic for fixed effects, in a static panel 
data model, under non-normality and fixed time periods. To the best of our knowledge this result is new. Interestingly, it has been shown that the commonly cited F-test procedure remains asymptotically valid. The second contribution of this paper is to uncover the asymptotic relationship between the F-test and the RE-test statistics. Under local (pure) random effects, they share the same asymptotic power, whilst under local fixed (or correlated) individual effects the $F$-test enjoys higher asymptotic power.

To assess the efficacy of these theoretical results, Monte Carlo evidence has been presented which suggests that, even under non-normality of the errors, (i) the use of the traditional $F$ distribution does indeed provide an excellent guide to finite sample behaviour, under the null, even in quite small samples; and (ii) the F-test always rejects as least as often as the RE-test under both random and fixed effects, with higher rejection frequencies when the effects are correlated with the regressors.

These results will be of use to applied workers since they not only confirm the robustness of the standard F-test procedure to non-normality, even in small samples, but also imply that the F-Test can be employed as a general test for the presence of individual effects (fixed or random) without loss of power.

\section{References}

[1] Ali, M. M. \& S. C. Sharma (1996) Robustness to nonnormality of regression F-tests. Journal of Econometrics 71, 175-205.

[2] Baltagi, B.H., Chang, Y., \& Li, Q. (1992) Monte Carlo results on several new and existing tests for the error component model. Journal of Econometrics 54, 95-120.

[3] Belsley, D. A., E. Kuh \& R. E. Welsch (1980) Regression Diagnostics. New York: John Wiley \& Sons.

[4] Beran, R. (1988) Prepivoting test statistics: A bootstrap view of asymptotic refinements. Journal of the American Statistical Association 83, 687-697.

[5] Box, G.E.P. \& S. L. Andersen (1955) Permutation theory in the derivation of robust criteria and the study of departures from assumptions. Journal of the Royal Statistical Society: Series B 17, 1-34.

[6] Breusch, T. S. \& A. R. Pagan (1980) The Lagrange multiplier test and its applications to model misspecification in econometrics. Review of Economic Studies 47, 239-253. 
[7] Chesher, A. D. (1984) Testing for neglected heterogeneity. Econometrica 52, 865-872.

[8] Godfrey, L. G. \& C. D. Orme (2000) Controlling the significance levels of prediction error tests for linear regression models. Econometrics Journal 3, 66-83.

[9] Honda, Y. (1985) Testing the error components model with non-normal disturbances. Review of Economic Studies 52, 681-690.

[10] Moulton, B.R., \& Randolph, W.C. (1989). Alternative tests of the error components model. Econometrica 57, 685-693.

[11] Nerlove, M. (1971) Further evidence on the estimation of dynamic economic relations from a time-series of cross-sections. Econometrica 39, 359-382.

[12] Qin, H. \& A.T.K Wan (2004) On the properties of the $t$ - and F-ratios in linear regressions with nonnormal errors. Econometric Theory 20, 690-700.

[13] Scheffe, H. (1959) The Analysis of Variance. New York: John Wiley \& Sons.

[14] White, H. (2001) Asymptotic Theory for Econometricians. Revised Edition. Academic Press.

[15] Wooldridge, J. M. (2002) Econometric Analysis of Cross Section and Panel Data. MIT Press.

[16] Yamagata, T. (2004) Essays on the Application of the Bootstrap to Improve Inference in Microeconometrics. Unpublished Ph.D. Thesis. University of Manchester. 


\section{APPENDIX}

\section{Proof of Proposition 1.}

Define $F_{N}=\frac{S_{N}}{\tilde{\sigma}^{2}}$, where $S_{N}=\left(R S S_{R}-R S S_{U}\right) /(N-1)$, so that

$$
\begin{aligned}
\sigma^{2} \sqrt{N}\left(F_{N}-1\right) & =\frac{\sigma^{2}}{\tilde{\sigma}^{2}} \sqrt{N}\left(S_{N}-\tilde{\sigma}^{2}\right) \\
& =\sqrt{N}\left(S_{N}-\tilde{\sigma}^{2}\right)+o_{p}(1)
\end{aligned}
$$

because $\tilde{\sigma}^{2}-\sigma^{2}=o_{p}(1)$. We first show that

$$
\sqrt{N}\left(S_{N}-\tilde{\sigma}^{2}\right)=\frac{1}{\sqrt{N}} \frac{\mathbf{u}^{\prime}\left(\mathbf{I}_{N} \otimes \mathbf{A}\right) \mathbf{u}}{(T-1)}+\lambda_{N}+o_{p}(1) .
$$

By the Frisch-Waugh theorem, $R S S_{U}$ is identical to that obtained from the ordinary least squares regression of $\tilde{\mathbf{y}}$ on $\tilde{\mathbf{X}}$, so that

$$
\begin{aligned}
R S S_{U} & =\mathbf{u}^{\prime} \mathbf{M}_{\mathbf{D}} \mathbf{M}_{\tilde{\mathbf{X}}} \mathbf{M}_{\mathbf{D}} \mathbf{u} \\
& =\mathbf{u}^{\prime}\left(\mathbf{M}_{\tilde{\mathbf{X}}}-\mathbf{P}_{\mathbf{D}}\right) \mathbf{u},
\end{aligned}
$$

since $\mathbf{M}_{\mathbf{D}} \mathbf{M}_{\tilde{\mathbf{X}}} \mathbf{M}_{\mathbf{D}}=\mathbf{M}_{\mathbf{D}}\left(\mathbf{M}_{\tilde{\mathbf{X}}}-\mathbf{P}_{\mathbf{D}}\right)=\mathbf{M}_{\tilde{\mathbf{X}}}-\mathbf{P}_{\mathbf{D}}$, so that $\tilde{\sigma}^{2}=\frac{1}{N(T-1)-K}\left[\mathbf{u}^{\prime}\left(\mathbf{M}_{\tilde{\mathbf{X}}}-\mathbf{P}_{\mathbf{D}}\right) \mathbf{u}\right]$. However, substituting for $\mathbf{y}$ from (1) and using Assumption $4(i)$ which states that $\boldsymbol{\alpha}=\beta_{0} \iota_{N}+N^{-1 / 4} \boldsymbol{\delta}$,

$$
\begin{aligned}
R S S_{R} & =\mathbf{y}^{\prime} \mathbf{M}_{\mathbf{Z}} \mathbf{y} \\
& =\mathbf{u}^{\prime} \mathbf{M}_{\mathbf{Z}} \mathbf{u}+\frac{2}{N^{1 / 4}} \mathbf{u}^{\prime} \mathbf{M}_{\mathbf{Z}} \mathbf{D} \boldsymbol{\delta}+\frac{1}{\sqrt{N}} \boldsymbol{\delta}^{\prime} \mathbf{D}^{\prime} \mathbf{M}_{\mathbf{Z}} \mathbf{D} \boldsymbol{\delta} .
\end{aligned}
$$

Note that this follows from the fact that $\mathbf{M}_{\mathbf{Z}} \mathbf{D} \boldsymbol{\alpha}=\mathbf{M}_{\mathbf{Z}} \boldsymbol{\delta}$, since $\mathbf{M}_{\mathbf{Z}} \mathbf{D} \boldsymbol{\iota}_{N}=$ $\mathbf{M}_{\mathbf{Z}} \iota_{N T} \equiv \mathbf{0}$. Thus, $S_{N}-\tilde{\sigma}^{2}$ can be expressed as

$$
\begin{aligned}
S_{N}-\tilde{\sigma}^{2} & =-\frac{\mathbf{u}^{\prime} \mathbf{P}_{\mathbf{Z}} \mathbf{u}}{N-1}+\frac{\mathbf{u}^{\prime} \mathbf{P}_{\tilde{\mathbf{X}}} \mathbf{u}}{N-1}+\frac{\mathbf{u}^{\prime} \mathbf{P}_{\mathbf{D}} \mathbf{u}}{N-1}+\frac{2}{N^{1 / 4}} \frac{\mathbf{u}^{\prime} \mathbf{M}_{\mathbf{Z}} \mathbf{D} \boldsymbol{\delta}}{N-1}+\frac{1}{\sqrt{N}} \frac{\boldsymbol{\delta}^{\prime} \mathbf{D}^{\prime} \mathbf{M}_{\mathbf{Z}} \mathbf{D} \boldsymbol{\delta}}{N-1} \\
& -\frac{N}{N(T-1)-K}\left[\frac{\mathbf{u}^{\prime} \mathbf{u}}{N}-\frac{\mathbf{u}^{\prime} \mathbf{P}_{\tilde{\mathbf{X}}} \mathbf{u}}{N}-\frac{\mathbf{u}^{\prime} \mathbf{P}_{\mathbf{D} \mathbf{u}}}{N}\right] \\
& =\frac{\mathbf{u}^{\prime} \mathbf{P}_{\mathbf{D}} \mathbf{u}}{N-1}-\frac{1}{T-1} \frac{\mathbf{u}^{\prime} \mathbf{u}}{N}+\frac{1}{T-1} \frac{\mathbf{u}^{\prime} \mathbf{P}_{\mathbf{D}} \mathbf{u}}{N}+\frac{1}{\sqrt{N}} \frac{\boldsymbol{\delta}^{\prime} \mathbf{D}^{\prime} \mathbf{M}_{\mathbf{Z}} \mathbf{D} \boldsymbol{\delta}}{N-1}+o_{p}\left(N^{-1 / 2}\right) \\
& =\frac{1}{N T} \sum_{i=1}^{N} v_{i}^{2}-\frac{1}{N(T-1)} \sum_{i=1}^{N} \sum_{t=1}^{T} u_{i t}^{2}+\frac{1}{N T(T-1)} \sum_{i=1}^{N} v_{i}^{2} \\
& +\frac{1}{\sqrt{N}} \frac{\boldsymbol{\delta}^{\prime} \mathbf{D}^{\prime} \mathbf{M}_{\mathbf{Z}} \mathbf{D} \boldsymbol{\delta}}{N-1}+o_{p}\left(N^{-1 / 2}\right) \\
& =\frac{1}{N(T-1)}\left(\sum_{i=1}^{N} v_{i}^{2}-\sum_{i=1}^{N} \sum_{t=1}^{T} u_{i t}^{2}\right)+\frac{1}{\sqrt{N}} \frac{\boldsymbol{\delta}^{\prime} \mathbf{D}^{\prime} \mathbf{M}_{\mathbf{Z}} \mathbf{D} \boldsymbol{\delta}}{N-1}+o_{p}\left(N^{-1 / 2}\right) \\
& =\frac{\mathbf{u}^{\prime}\left(\mathbf{I}_{N} \otimes \mathbf{A}\right) \mathbf{u}}{N(T-1)}+\frac{1}{\sqrt{N}} \lambda_{N}+o_{p}\left(N^{-1 / 2}\right)
\end{aligned}
$$


recalling that $v_{i}=\sum_{t=1}^{T} u_{i t}$. The second equality follows because (i) both $\mathbf{u}^{\prime} \mathbf{P}_{\mathbf{Z}} \mathbf{u}$ and $\mathbf{u}^{\prime} \mathbf{P}_{\tilde{\mathbf{X}}} \mathbf{u}$ are $O_{p}(1)$, under Assumptions 1 and 2 ; (ii) $\frac{N}{N(T-1)-K}=$ $\frac{1}{(T-1)}+O\left(N^{-1}\right)$; (iii) $(N-1)^{-1} \mathbf{u}^{\prime} \mathbf{D} \boldsymbol{\delta}=(N-1)^{-1} \sum_{i=1}^{N} v_{i} \delta_{i}=O_{p}\left(N^{-1 / 2}\right)$, under Assumptions 1(i),1(ii),2(i) and 4(ii), 4(iii) which ensure that a Central Limit Theorem holds for $N^{-1 / 2} \sum_{i=1}^{N} v_{i} \delta_{i}$; and, (iv) $\frac{\mathbf{Z}^{\prime} \mathbf{D} \boldsymbol{\delta}}{N}=\frac{T}{N} \sum_{i=1}^{N} \delta_{i} \overline{\mathbf{z}}_{i}=$ $O_{p}(1)$, at most, under Assumptions 2(ii) and 4(ii) which ensure that a Law of Large Numbers applies to $\frac{1}{N} \sum_{i=1}^{N} \delta_{i} \overline{\mathbf{z}}_{i}$, since by Chebsyshev's Inequality and $\eta>0$

$$
E\left|\delta_{i} \overline{\mathbf{z}}_{i}\right|^{1+\eta} \leq \sqrt{E\left|\delta_{i}\right|^{2+2 \eta} E\left|\overline{\mathbf{z}}_{i}\right|^{2+2 \eta}} \leq \Delta<\infty .
$$

The third equality uses $\mathbf{u}^{\prime} \mathbf{P}_{\mathbf{D}} \mathbf{u}=\frac{1}{T} \sum_{i=1}^{N}\left(\sum_{t=1}^{T} u_{i t}\right)^{2}=O_{p}(N)$, so that

$$
\begin{aligned}
\frac{\mathbf{u}^{\prime} \mathbf{P}_{\mathbf{D}} \mathbf{u}}{N-1} & =\frac{1}{(N-1) T} \sum_{i=1}^{N} v_{i}^{2} \\
& =\frac{1}{N T} \sum_{i=1}^{N} v_{i}^{2}+\frac{1}{N(N-1) T} \sum_{i=1}^{N} v_{i}^{2} \\
& =\frac{1}{N T} \sum_{i=1}^{N} v_{i}^{2}+O_{p}\left(N^{-1}\right) .
\end{aligned}
$$

The fifth equality follows from the fact that

$$
\begin{aligned}
\lambda_{N} & =\frac{\boldsymbol{\delta}^{\prime} \mathbf{D}^{\prime} \mathbf{M}_{\mathbf{Z}} \mathbf{D} \boldsymbol{\delta}}{N} \\
& =T\left\{\frac{1}{N} \sum_{i=1}^{N} \delta_{i}^{2}-T\left(\frac{1}{N} \sum_{i=1}^{N} \delta_{i} \overline{\mathbf{z}}_{i}^{\prime}\right)\left(\frac{\mathbf{Z}^{\prime} \mathbf{Z}}{N}\right)^{-1}\left(\frac{1}{N} \sum_{i=1}^{N} \delta_{i} \overline{\mathbf{z}}_{i}^{\prime}\right)\right\} \\
& =O_{p}(1)
\end{aligned}
$$

by Assumptions 2(ii), 2(iii) and 4(ii),4(iii), so that $\frac{\boldsymbol{\delta}^{\prime} \mathbf{D}^{\prime} \mathbf{M}_{\mathbf{Z}} \mathbf{D} \boldsymbol{\delta}}{N-1}=\lambda_{N}+$ $O_{p}\left(N^{-1}\right)$, and the fact that $\mathbf{u}^{\prime}\left(\mathbf{I}_{N} \otimes \mathbf{A}\right) \mathbf{u}=\sum_{i=1}^{N} v_{i}^{2}-\sum_{i=1}^{N} \sum_{t=1}^{T} u_{i t}^{2}$.

Second, to establish the limit distribution, write

$$
\sqrt{N}\left(S_{N}-\tilde{\sigma}^{2}\right)=\frac{1}{(T-1) \sqrt{N}} \sum_{i=1}^{N} W_{i}+\lambda_{N}+o_{p}(1)
$$

where the $W_{i}=\mathbf{u}_{i}^{\prime} \mathbf{A} \mathbf{u}_{i}$ are independent, by Assumption 1(i). It is easy to determine that $E\left(W_{i}\right)=0$, since the $u_{i t}$ are serially uncorrelated by Assumption 1(iii). Since the elements of $\left\{u_{i t}^{2}\right\}$ are also serially uncorrelated by Assumption 3(i), a little calculation reveals that (because the diagonal elements of $\mathbf{A}$ are zero)

$$
\begin{aligned}
\operatorname{var}\left(W_{i}\right) & =2 \sigma^{4} \operatorname{tr}\left(\mathbf{A}^{2}\right) \\
& =2 \sigma^{4} T(T-1)<\infty
\end{aligned}
$$


where $\operatorname{tr}$ (.) signifies trace. Finally, since $\left|W_{i}\right|=\left|\mathbf{u}_{i}^{\prime} \mathbf{A} \mathbf{u}_{i}\right| \leq\left|\mathbf{u}_{i}\right|^{2}\|\mathbf{A}\|$, where $\left|\mathbf{u}_{i}\right|^{2}=\sum_{t=1}^{T} u_{i t}^{2}$ and $\|\mathbf{A}\|=\sqrt{\operatorname{tr}\left(\mathbf{A}^{\prime} \mathbf{A}\right)}=\sqrt{T(T-1)}$, we have that for some $\eta>0$,

$$
\begin{aligned}
E\left|W_{i}\right|^{2+\eta} & \leq(T(T-1))^{1+\eta / 2} E\left|\sum_{t=1}^{T} u_{i t}^{2}\right|^{2+\eta} \\
& \leq(T(T-1))^{1+\eta / 2}\left[\sum_{t=1}^{T}\left\{E\left|u_{i t}^{2}\right|^{2+\eta}\right\}^{\frac{1}{2+\eta}}\right]^{2+\eta} \\
& \leq(T(T-1))^{1+\eta / 2}\left[T \Delta^{\frac{1}{2+\eta}}\right]^{2+\eta} \\
& =(T(T-1))^{1+\eta / 2} T^{2+\eta} \Delta=O(1) .
\end{aligned}
$$

Thus, a standard (Liapounov) Central Limit Theorem yields, $\sqrt{N}\left(S_{N}-\tilde{\sigma}^{2}\right)-$ $\lambda_{N} \stackrel{d}{\rightarrow} N\left(0, \frac{2 \sigma^{4} T}{T-1}\right)$, and the result follows, which completes the proof.

Proof of Proposition 2

We can write $R_{N}=\left\{\sqrt{\frac{1}{2 T(T-1)}}\right\} \frac{H_{N}}{\hat{\sigma}^{2}}$, where $\hat{\sigma}^{2}=\hat{\mathbf{u}}^{\prime} \hat{\mathbf{u}} / N T$ and

$$
\begin{aligned}
H_{N} & =\frac{1}{\sqrt{N}}\left[\hat{\mathbf{u}}^{\prime}\left(\mathbf{I}_{N} \otimes \mathbf{A}\right) \hat{\mathbf{u}}\right] \\
& =\frac{1}{\sqrt{N}}\left[\mathbf{y}^{\prime} \mathbf{M}_{\mathbf{Z}}\left(\mathbf{I}_{N} \otimes \mathbf{A}\right) \mathbf{M}_{\mathbf{Z}} \mathbf{y}\right]
\end{aligned}
$$

We show that

$$
\begin{aligned}
H_{N} & =\frac{1}{\sqrt{N}}\left[\mathbf{u}^{\prime}\left(\mathbf{I}_{N} \otimes \mathbf{A}\right) \mathbf{u}\right]+(T-1) \lambda_{N}-T \gamma_{N}+o_{p}(1) \\
\hat{\sigma}^{2} & =\sigma^{2}+o_{p}(1)
\end{aligned}
$$

and the result follows, from Proposition 1.

Making the substitution of $\mathbf{M}_{\mathbf{Z}} \mathbf{y}=N^{-1 / 4} \mathbf{M}_{\mathbf{Z}} \mathbf{D} \boldsymbol{\delta}+\mathbf{M}_{\mathbf{Z}} \mathbf{u}$, under Assumption $4(i)$ as before, we have

$$
\begin{aligned}
H_{N}= & \frac{1}{\sqrt{N}}\left[\mathbf{u}^{\prime}\left(\mathbf{I}_{N} \otimes \mathbf{A}\right) \mathbf{u}\right]+\frac{1}{N}\left[\boldsymbol{\delta}^{\prime} \mathbf{D}^{\prime} \mathbf{M}_{\mathbf{Z}}\left(\mathbf{I}_{N} \otimes \mathbf{A}\right) \mathbf{M}_{\mathbf{Z}} \mathbf{D} \boldsymbol{\delta}\right] \\
& +\frac{1}{\sqrt{N}}\left[\mathbf{u}^{\prime} \mathbf{P}_{\mathbf{Z}}\left(\mathbf{I}_{N} \otimes \mathbf{A}\right) \mathbf{P} \mathbf{Z} \mathbf{u}\right] \\
& -\frac{2}{\sqrt{N}}\left[\mathbf{u}^{\prime}\left(\mathbf{I}_{N} \otimes \mathbf{A}\right) \mathbf{P}_{\mathbf{Z}} \mathbf{u}\right]+\frac{2}{N^{3 / 4}}\left[\mathbf{u}^{\prime} \mathbf{M}_{\mathbf{Z}}\left(\mathbf{I}_{N} \otimes \mathbf{A}\right) \mathbf{M}_{\mathbf{Z}} \mathbf{D} \boldsymbol{\delta}\right] \\
= & \sum_{j=0}^{4} V_{j N}
\end{aligned}
$$


The first term, $V_{0 N}=\frac{1}{\sqrt{N}}\left[\mathbf{u}^{\prime}\left(\mathbf{I}_{N} \otimes \mathbf{A}\right) \mathbf{u}\right]$ is $O_{p}(1)$, by Proposition 1, and we show that $V_{1 N}=(T-1) \lambda_{N}-T \gamma_{N}+o_{p}(1)$, whilst $V_{j N}=o_{p}(1), j=1,2,3,4$.

Before proceeding, note the following results:

1. $\frac{\tilde{\mathbf{Z}}^{\prime} \tilde{\mathbf{Z}}}{N}=\frac{\mathbf{Z}^{\prime} \mathbf{Z}}{N}-\frac{T}{N} \overline{\mathbf{Z}}^{\prime} \overline{\mathbf{Z}}$, where $\overline{\mathbf{Z}},(N \times K+1)$, has rows $\overline{\mathbf{z}}_{i}^{\prime}=T^{-1} \sum_{t=1}^{T} \mathbf{z}_{i t}^{\prime}$.

2.

$$
\begin{aligned}
\frac{\mathbf{Z}^{\prime}\left(\mathbf{I}_{N} \otimes \mathbf{A}\right) \mathbf{Z}}{N} & =\frac{1}{N}\left\{\sum_{i=1}^{N}\left(\sum_{t=1}^{T} \mathbf{z}_{i t}\right)\left(\sum_{t=1}^{T} \mathbf{z}_{i t}^{\prime}\right)-\sum_{i=1}^{N} \sum_{t=1}^{T} \mathbf{z}_{i t} \mathbf{z}_{i t}^{\prime}\right\} \\
& =T^{2} \frac{\overline{\mathbf{Z}}^{\prime} \overline{\mathbf{Z}}}{N}-\frac{\mathbf{Z}^{\prime} \mathbf{Z}}{N} \\
& =(T-1)\left(\frac{\mathbf{Z}^{\prime} \mathbf{Z}}{N}\right)-T\left(\frac{\tilde{\mathbf{Z}} \cdot \tilde{\mathbf{Z}}}{N}\right) \\
& =O_{p}(1)
\end{aligned}
$$

3. $\mathbf{Z}^{\prime}\left(\mathbf{I}_{N} \otimes \mathbf{A}\right) \mathbf{D} \boldsymbol{\delta}=(T-1) \mathbf{Z}^{\prime} \mathbf{D} \boldsymbol{\delta}=T(T-1) \sum_{i=1}^{N} \mathbf{z}_{i} \delta_{i}$.

4. $\boldsymbol{\delta}^{\prime} \mathbf{D}^{\prime}\left(\mathbf{I}_{N} \otimes \mathbf{A}\right) \mathbf{D} \boldsymbol{\delta}=(T-1) \boldsymbol{\delta}^{\prime} \mathbf{D}^{\prime} \mathbf{D} \boldsymbol{\delta}=T(T-1) \sum_{i=1}^{N} \delta_{i}^{2}$.

Then,

$$
\begin{aligned}
V_{1 N}= & \frac{1}{N}\left[\boldsymbol{\delta}^{\prime} \mathbf{D}^{\prime}\left(\mathbf{I}_{N} \otimes \mathbf{A}\right) \mathbf{D} \boldsymbol{\delta}-\boldsymbol{\delta}^{\prime} \mathbf{D}^{\prime} \mathbf{P}_{\mathbf{Z}}\left(\mathbf{I}_{N} \otimes \mathbf{A}\right) \mathbf{D} \boldsymbol{\delta}\right. \\
& \left.-\boldsymbol{\delta}^{\prime} \mathbf{D}^{\prime}\left(\mathbf{I}_{N} \otimes \mathbf{A}\right) \mathbf{P}_{\mathbf{Z}} \mathbf{D} \boldsymbol{\delta}+\boldsymbol{\delta}^{\prime} \mathbf{D}^{\prime} \mathbf{P}_{\mathbf{Z}}\left(\mathbf{I}_{N} \otimes \mathbf{A}\right) \mathbf{P}_{\mathbf{Z}} \mathbf{D} \boldsymbol{\delta}\right] \\
= & {\left[\frac{T(T-1)}{N} \sum_{i=1}^{N} \delta_{i}^{2}-2(T-1) \frac{\boldsymbol{\delta}^{\prime} \mathbf{D}^{\prime} \mathbf{Z}}{N}\left(\frac{\mathbf{Z}^{\prime} \mathbf{Z}}{N}\right)^{-1} \frac{\mathbf{Z}^{\prime} \mathbf{D} \boldsymbol{\delta}}{N}\right.} \\
& \left.+\frac{\boldsymbol{\delta}^{\prime} \mathbf{D}^{\prime} \mathbf{Z}}{N}\left(\frac{\mathbf{Z}^{\prime} \mathbf{Z}}{N}\right)^{-1}\left\{(T-1)\left(\frac{\mathbf{Z}^{\prime} \mathbf{Z}}{N}\right)-T\left(\frac{\tilde{\mathbf{Z}}}{N}\right)\right\}\left(\frac{\mathbf{Z}^{\prime} \mathbf{Z}}{N}\right)^{-1} \frac{\mathbf{Z}^{\prime} \mathbf{D} \boldsymbol{\delta}}{N}\right] \\
= & (T-1) \frac{\boldsymbol{\delta}^{\prime} \mathbf{D}^{\prime} \mathbf{M}_{\mathbf{Z}} \mathbf{D} \boldsymbol{\delta}}{N}-T \frac{\boldsymbol{\delta}^{\prime} \mathbf{D}^{\prime} \mathbf{Z}}{N}\left(\frac{\mathbf{Z}^{\prime} \mathbf{Z}}{N}\right)^{-1}\left(\frac{\tilde{\mathbf{Z}}}{N} \tilde{\mathbf{Z}}\right)\left(\frac{\mathbf{Z}^{\prime} \mathbf{Z}}{N}\right)^{-1} \frac{\mathbf{Z}^{\prime} \mathbf{D} \boldsymbol{\delta}}{N} \\
= & (T-1) \lambda_{N}-T \gamma_{N} .
\end{aligned}
$$

Noting that

$$
\frac{\boldsymbol{\delta}^{\prime} \mathbf{D}^{\prime} \mathbf{M}_{\mathbf{Z}} \mathbf{D} \boldsymbol{\delta}}{N}=\frac{T}{N} \sum_{i=1}^{N} \delta_{i}^{2}-\frac{\boldsymbol{\delta}^{\prime} \mathbf{D}^{\prime} \mathbf{Z}}{N}\left(\frac{\mathbf{Z}^{\prime} \mathbf{Z}}{N}\right)^{-1} \frac{\mathbf{Z}^{\prime} \mathbf{D} \boldsymbol{\delta}}{N}=O_{p}(1) .
$$




$$
\begin{aligned}
V_{2 N} & =\frac{1}{\sqrt{N}}\left[\mathbf{u}^{\prime} \mathbf{Z}\left(\mathbf{Z}^{\prime} \mathbf{Z}\right)^{-1} \mathbf{Z}^{\prime}\left(\mathbf{I}_{N} \otimes \mathbf{A}\right) \mathbf{Z}\left(\mathbf{Z}^{\prime} \mathbf{Z}\right)^{-1} \mathbf{Z}^{\prime} \mathbf{u}\right] \\
& =\frac{1}{\sqrt{N}}\left[\frac{\mathbf{u}^{\prime} \mathbf{Z}}{\sqrt{N}}\left(\frac{\mathbf{Z}^{\prime} \mathbf{Z}}{N}\right)^{-1}\left\{\frac{\mathbf{Z}^{\prime}\left(\mathbf{I}_{N} \otimes \mathbf{A}\right) \mathbf{Z}}{N}\right\}\left(\frac{\mathbf{Z}^{\prime} \mathbf{Z}}{N}\right)^{-1} \frac{\mathbf{Z}^{\prime} \mathbf{u}}{\sqrt{N}}\right] \\
& =O_{p}\left(N^{-1 / 2}\right) . \\
V_{3 N} & =\frac{2}{\sqrt{N}}\left[\mathbf{u}^{\prime}\left(\mathbf{I}_{N} \otimes \mathbf{A}\right) \mathbf{Z}\left(\mathbf{Z}^{\prime} \mathbf{Z}\right)^{-1} \mathbf{Z}^{\prime} \mathbf{u}\right] \\
& =2\left[\left\{\frac{\mathbf{u}^{\prime}\left(\mathbf{I}_{N} \otimes \mathbf{A}\right) \mathbf{Z}}{N}\right\}\left(\frac{\mathbf{Z}^{\prime} \mathbf{Z}}{N}\right)^{-1} \frac{\mathbf{Z}^{\prime} \mathbf{u}}{\sqrt{N}}\right] \\
& =-2\left[\left\{\frac{1}{N} \sum_{i=1}^{N} \sum_{t=1}^{T} u_{i t} \mathbf{z}_{i t}^{\prime}-\frac{T}{N} \sum_{i=1}^{N} v_{i} \overline{\mathbf{z}}_{i}^{\prime}\right\}\left(\frac{\mathbf{Z}^{\prime} \mathbf{Z}}{N}\right)^{-1} \frac{\mathbf{Z}^{\prime} \mathbf{u}}{\sqrt{N}}\right] \\
& =O_{p}\left(N^{-1 / 2}\right)
\end{aligned}
$$

because, under Assumptions 1 and 2 , both $\frac{1}{N} \sum_{i=1}^{N} \sum_{t=1}^{T} u_{i t} \mathbf{z}_{i t}^{\prime}$ and $\frac{T}{N} \sum_{i=1}^{N} v_{i} \overline{\mathbf{z}}_{i}^{\prime}$ are $O_{p}\left(N^{-1 / 2}\right)$.

$$
\begin{aligned}
V_{4 N}= & \frac{2}{N^{3 / 4}}\left[\mathbf{u}^{\prime}\left(\mathbf{I}_{N} \otimes \mathbf{A}\right) \mathbf{D} \boldsymbol{\delta}-\mathbf{u}^{\prime} \mathbf{P}_{\mathbf{Z}}\left(\mathbf{I}_{N} \otimes \mathbf{A}\right) \mathbf{D} \boldsymbol{\delta}\right. \\
& \left.-\mathbf{u}^{\prime}\left(\mathbf{I}_{N} \otimes \mathbf{A}\right) \mathbf{P}_{\mathbf{Z}} \mathbf{D} \boldsymbol{\delta}+\mathbf{u}^{\prime} \mathbf{P}_{\mathbf{Z}}\left(\mathbf{I}_{N} \otimes \mathbf{A}\right) \mathbf{P}_{\mathbf{Z}} \mathbf{D} \boldsymbol{\delta}\right] \\
= & \frac{2}{N^{1 / 4}}\left[(T-1) \frac{1}{\sqrt{N}} \sum_{i=1}^{N} v_{i} \delta_{i}-(T-1) \frac{\mathbf{u}^{\prime} \mathbf{Z}}{\sqrt{N}}\left(\frac{\mathbf{Z}^{\prime} \mathbf{Z}}{N}\right)^{-1} \frac{\mathbf{Z}^{\prime} \mathbf{D} \boldsymbol{\delta}}{N}\right. \\
& -\sqrt{N}\left\{\frac{\mathbf{u}^{\prime}\left(\mathbf{I}_{N} \otimes \mathbf{A}\right) \mathbf{Z}}{N}\right\}\left(\frac{\mathbf{Z}^{\prime} \mathbf{Z}}{N}\right)^{-1} \frac{\mathbf{Z}^{\prime} \mathbf{D} \boldsymbol{\delta}}{N} \\
& \left.+\frac{\mathbf{u}^{\prime} \mathbf{Z}}{\sqrt{N}}\left(\frac{\mathbf{Z}^{\prime} \mathbf{Z}}{N}\right)^{-1}\left\{\frac{\mathbf{Z}^{\prime}\left(\mathbf{I}_{N} \otimes \mathbf{A}\right) \mathbf{Z}}{N}\right\}\left(\frac{\mathbf{Z}^{\prime} \mathbf{Z}}{N}\right)^{-1} \frac{\mathbf{Z}^{\prime} \mathbf{D} \boldsymbol{\delta}}{N}\right] \\
= & O_{p}\left(N^{-1 / 4}\right),
\end{aligned}
$$

Finally, and using previous results,

$$
\begin{aligned}
\hat{\sigma}^{2} & =\frac{\mathbf{y}^{\prime} \mathbf{M}_{\mathbf{Z}} \mathbf{y}}{N T} \\
& =N^{-1 / 2} \frac{\boldsymbol{\delta}^{\prime} \mathbf{D}^{\prime} \mathbf{M}_{\mathbf{Z}} \mathbf{D} \boldsymbol{\delta}}{N T}+2 N^{-1 / 4} \frac{\boldsymbol{\delta}^{\prime} \mathbf{D}^{\prime} \mathbf{M}_{\mathbf{Z}} \mathbf{u}}{N T}+\frac{\mathbf{u}^{\prime} \mathbf{M}_{\mathbf{Z}} \mathbf{u}}{N T} \\
& =\frac{\mathbf{u}^{\prime} \mathbf{u}}{N T}+O_{p}\left(N^{-1 / 4}\right) \\
& =\sigma^{2}+o_{p}(1) .
\end{aligned}
$$

This completes the proof 
Table 1: Leverage Points

\begin{tabular}{|c|c|c|c|c|}
\hline & $N=$ & 20 & 50 & 100 \\
\hline The maximum value of $h_{s} / \bar{h}$ & & 5.4 & 5.7 & 5.3 \\
\hline The number of leverage points & & 5 & 16 & 30 \\
\hline
\end{tabular}

Table 2:Estimated Size of the Tests at 5\% Level

\begin{tabular}{|c|c|c|c|c|c|c|c|c|c|c|}
\hline \multirow[b]{2}{*}{ errors } & \multirow[b]{2}{*}{$N=$} & \multicolumn{3}{|c|}{$F_{N}$} & \multicolumn{3}{|c|}{$F_{N}^{*}$} & \multicolumn{3}{|c|}{$R_{N}$} \\
\hline & & 20 & 50 & 100 & 20 & 50 & 100 & 20 & 50 & 100 \\
\hline$S N$ & & 5.48 & 4.98 & 4.88 & $\underline{8.62}$ & 7.16 & $\underline{6.10}$ & 4.72 & 4.56 & 4.64 \\
\hline$t(5)$ & & 4.70 & 4.48 & 5.38 & $\overline{\overline{7.94}}$ & $\underline{\underline{6.58}}$ & $\underline{\underline{6.88}}$ & $\underline{4.00}$ & 4.20 & 4.98 \\
\hline$U N$ & & 5.24 & 4.64 & 5.34 & $\overline{8.50}$ & $\underline{6.56}$ & $\underline{6.88}$ & 4.52 & 4.36 & 5.14 \\
\hline$M N$ & & 5.20 & 5.06 & 5.42 & $\underline{\overline{8.58}}$ & $\overline{\overline{7.30}}$ & $\overline{7.28}$ & 4.38 & 4.68 & 4.98 \\
\hline$L N$ & & 4.28 & 5.36 & 5.70 & $\begin{array}{l}\overline{7.06} \\
\end{array}$ & $\overline{\overline{7.22}}$ & $\underline{\underline{6.60}}$ & $\underline{3.82}$ & 5.04 & 5.40 \\
\hline$\chi^{2}(2)$ & & 5.06 & 4.70 & 5.48 & $\underline{\overline{8.06}}$ & $\overline{\overline{6.46}}$ & $\overline{\underline{6.88}}$ & 4.24 & 4.34 & 5.04 \\
\hline
\end{tabular}

Notes:

Single (resp. double) underline denotes that the rejection frequency is not consistent with the true significance level being at most $0.5 \%$ (resp. 1\%) from the nominal level.

See Godfrey and Orme (2000). 
Table 3: Estimated Power of Tests at 5\% Level

\begin{tabular}{|c|c|c|c|c|c|c|c|c|c|}
\hline \multirow{2}{*}{\multicolumn{2}{|c|}{$\begin{array}{l}\text { Errors } \\
\text { Design }\end{array}$}} & \multicolumn{8}{|c|}{$S N$} \\
\hline & & \multicolumn{2}{|c|}{$F E 1$} & \multicolumn{2}{|c|}{$F E 2$} & \multicolumn{2}{|c|}{$R E 1$} & \multicolumn{2}{|c|}{$R E 2$} \\
\hline$\tau$ & $N$ & $F_{N}$ & $R_{N}$ & $F_{N}$ & $R_{N}$ & $\overline{F_{N}}$ & $R_{N}$ & $F_{N}$ & $R_{N}$ \\
\hline 0.05 & 20 & 15.84 & 12.48 & 11.34 & 10.16 & 19.82 & 14.78 & 14.90 & 13.56 \\
\hline 0.05 & 50 & 2.34 & 3 & 42.34 & 40.70 & 28.72 & 23.82 & 26.70 & 25.30 \\
\hline 0.05 & 100 & 52.46 & 46.60 & 52.04 & 50.76 & 44.52 & 39.28 & 42.74 & 41.52 \\
\hline 0.1 & 20 & 32.28 & 24.82 & 19.70 & 18.04 & 40.24 & 31.02 & 29.38 & 27.06 \\
\hline 0.1 & 50 & 81.56 & 73.98 & 81.32 & 80.42 & 62.60 & 54.54 & 57.16 & 55.50 \\
\hline 0.1 & 100 & 91.68 & 88.22 & 91.08 & 90.74 & 84.98 & 79.68 & 82.00 & 81.42 \\
\hline 0.2 & 20 & 66.46 & 56.38 & 42.22 & 40.26 & 75.86 & 65.92 & 58.50 & 55.78 \\
\hline 0.2 & 50 & 99.66 & 9.04 & 9.42 & 99.40 & 95.30 & 91.50 & 91.38 & 90.86 \\
\hline 0.2 & 100 & 100.00 & 99.94 & 100.00 & 100.00 & 99.78 & 99.60 & 99.70 & 99.70 \\
\hline \multicolumn{2}{|c|}{ Errors } & \multicolumn{8}{|c|}{$M N$} \\
\hline \multicolumn{2}{|c|}{ Design } & \multicolumn{2}{|c|}{$F E 1$} & \multicolumn{2}{|c|}{$F E 2$} & \multicolumn{2}{|c|}{$R E 1$} & \multicolumn{2}{|c|}{$R E 2$} \\
\hline$\tau$ & $N$ & $F_{N}$ & $R_{N}$ & $F_{N}$ & $R_{N}$ & $F_{N}$ & $R_{N}$ & $F_{N}$ & $R_{N}$ \\
\hline 0.05 & 20 & 16.16 & 12.54 & 11.90 & 10.62 & 19.84 & 15.64 & 15.88 & 14.44 \\
\hline 05 & 50 & .04 & 32.42 & 40.20 & 39.08 & 28.80 & 23.02 & 25.28 & 23.92 \\
\hline 0.05 & 100 & 52.28 & 46.86 & 51.32 & 50.58 & 43.30 & 38.38 & 41.12 & 39.90 \\
\hline 0.1 & 20 & 32.12 & 24.62 & 20.40 & 18.76 & 41.20 & 31.34 & 30.36 & 28.24 \\
\hline 0.1 & 50 & 0.72 & 72.34 & 80.74 & 80.06 & 61.76 & 53.48 & 55.52 & 53.92 \\
\hline 0.1 & 100 & 92.14 & 88.82 & 92.02 & 91.60 & 83.82 & 79.70 & 81.08 & 80.32 \\
\hline 0.2 & 20 & 66.08 & 55.62 & 42.22 & 40.32 & 75.48 & 65.94 & 58.78 & 56.44 \\
\hline 0.2 & 50 & .54 & & 99.58 & 99.54 & 95.80 & 92.26 & 91.32 & 90.80 \\
\hline 0.2 & 100 & 100.00 & 100.00 & 100.00 & 100.00 & 99.88 & 99.78 & 99.58 & 99.54 \\
\hline \multicolumn{2}{|c|}{ Errors } & \multicolumn{8}{|c|}{$\chi^{2}(2)$} \\
\hline \multicolumn{2}{|c|}{ Design } & \multicolumn{2}{|c|}{$F E 1$} & \multicolumn{2}{|c|}{$F E 2$} & \multicolumn{2}{|c|}{$R E 1$} & \multicolumn{2}{|c|}{$R E 2$} \\
\hline$\tau$ & $N$ & $F_{N}$ & $R_{N}$ & $F_{N}$ & $R_{N}$ & $F_{N}$ & $R_{N}$ & $F_{N}$ & $R_{N}$ \\
\hline 0.05 & 20 & 16.32 & 12.80 & 11.02 & 9.74 & 20.32 & 15.42 & 16.14 & 14.24 \\
\hline 0.05 & 50 & 2.38 & & 41.92 & 40.76 & 29.38 & 24.18 & 26.36 & 25.00 \\
\hline 0.05 & 100 & 51.86 & 45.76 & 51.38 & 50.30 & 42.78 & 37.60 & 40.92 & 39.66 \\
\hline 0.1 & 20 & 33.64 & 25.88 & 21.38 & 19.56 & 41.60 & 33.12 & 31.58 & 29.32 \\
\hline 0.1 & 50 & & 74.02 & 81.90 & 80.84 & 63.80 & 55.16 & 58.14 & 56.74 \\
\hline 0.1 & 100 & 92.04 & 88.32 & 91.32 & 91.18 & 84.40 & 79.78 & 81.12 & 80.66 \\
\hline 0.2 & 20 & 67.06 & 58.78 & 44.56 & 42.62 & 74.98 & 65.52 & 59.60 & 57.44 \\
\hline 0.2 & 50 & 9.22 & & 99.22 & 99.18 & 94.60 & 91.48 & 90.52 & 90.00 \\
\hline 0.2 & 100 & 99.98 & 99.96 & 99.96 & 99.96 & 99.64 & 99.34 & 99.28 & 99.24 \\
\hline
\end{tabular}

